\title{
ON THE NONLINEAR CONVEXITY THEOREM OF KOSTANT
}

\author{
JIANG-HUA LU AND TUDOR RATIU
}

\section{INTRODUCTION}

A classical result of Schur and Horn [Sc, Ho] states that the set of diagonal elements of all $n \times n$ Hermitian matrices with fixed eigenvalues is a convex set in $\mathbb{R}^{n}$. Kostant $[\mathrm{Kt}]$ has generalized this result to the case of any semisimple Lie group. This is often referred to as the linear convexity theorem of Kostant: picking up the diagonal of a Hermitian matrix is a linear operation. This result was later put into the framework of symplectic geometry by Atiyah [Ay], Guillemin-Sternberg [Gu-St] and Duistermaat [Du1], the key argument being that the map that picks up the diagonal of a Hermitian matrix is a moment map for a Hamiltonian torus action and that the image of such a moment map is always a convex polytope.

There is also a nonlinear version of the convexity theorem of Kostant. It has not been connected so far with symplectic geometry. The purpose of this paper is to make this connection.

We first describe the nonlinear convexity theorem for the case of $\operatorname{SL}(n, \mathbb{C})$ (see $\S 2$ for more details). The Gram-Schmidt orthonormalization process in linear algebra asserts that every nonsingular $n \times n$ complex matrix $g$ can be uniquely written as the product $g=k a n$ for some unitary matrix $k$, positive diagonal matrix $a$, and strictly upper-triangular matrix $n$. We use $A$ to denote the group of all positive diagonal matrices, and we call the matrix $a$ in the above decomposition the $A$-component of $g$. The nonlinear convexity theorem of Kostant now asserts that the set of $A$-components of $g$ as $g$ runs through all positive definite Hermitian matrices with fixed eigenvalues $a=\left(a_{1}, \ldots, a_{n}\right)$ is the convex hull of the set of all points obtained by permuting the coordinates of $a$. Here we identify $A$ with its Lie algebra a via the exponential map; thus convexity in $A$ makes sense.

For a general semisimple Lie group $G$, the Gram-Schmidt decomposition is replaced by an Iwasawa decomposition $G=K A N$ of $G$, and the permutation group that appeared above is replaced by the Weyl group $W$ of $(K, A)$. If an element $g \in G$ is written as the product $g=k a n$ for $k \in K, a \in A$,

Received by the editors November 21, 1990.

1991 Mathematics Subject Classification. Primary 22E10, 32M05.

The first author was partially supported by DOE contract DE-FG03-88ER25064. The second author was partially supported by NSF grant DMS-8922699. 
and $n \in N$, then $a$ is called the $A$-component of $g$. For any $a \in A$, the set of $A$-components of $k a k^{-1}$ as $k$ runs through $K$ often appears in the representation theory of $G$. Kostant's nonlinear convexity theorem now states that this set is the convex hull of the Weyl group orbit $W \cdot a$ in $A$.

We remark that as $a$ and $k$ respectively run through $A$ and $K$, the point $k a k^{-1}$ runs through the symmetric space $P$. (In the case when $G=\operatorname{SL}(n, \mathbb{C})$, $P$ is the space of all positive definite $n \times n$ Hermitian matrices with determinant 1.) Each set $\left\{k a k^{-1}: k \in K\right\}$ for fixed $a \in A$ is an orbit of the $K$-action on $P$ by conjugations. Such an orbit is usually called a flag manifold. When $G$ is a complex semisimple Lie group, $\operatorname{such}$ as $\operatorname{SL}(n, \mathbb{C})$, but considered as a real Lie group, these orbits are called complex flag manifolds. When $G$ is a real form of a complex semisimple Lie group, such as $\operatorname{SL}(n, \mathbb{R})$, these orbits are called real flag manifolds.

In this paper, we will show that when $G$ is a complex semisimple Lie group, there is a Poisson structure $\pi$ on the symmetric space $P$ whose symplectic leaves are exactly the $K$-orbits in $P$. The Poisson structure $\pi$ has the additional property that it is invariant under the action of a maximal torus $T$ of $K$, and, most importantly, the map that picks up the $A$-component of an element is an equivariant moment map for this torus action with respect to $\pi$. Applying the convexity theorem of Atiyah-Guillemin-Sternberg to each orbit, i.e., each symplectic leaf, we immediately obtain the nonlinear convexity theorem of Kostant.

The first step in defining $\pi$ is to identify $P$ with the subgroup $A N$ of $G$ via the two decompositions $G=K P$ (the Cartan decomposition) and $G=K A N$ (the Iwasawa decomposition) of $G$. We then define a Poisson structure on $A N$, which, when considered as defined on $P$, has the aforementioned properties.

Our result is a consequence of those in [Lu-We] on Poisson Lie groups. In fact, the Poisson structure on $A N$ that we are going to use is defined in [Lu-We] as an example of Poisson Lie groups. It has also been observed by Reshetikhin [Rs] and de Concini, Kac, and Procesi [Co-Kc] [C-K-P] that this Poisson structure plays an important role in the representation theory of quantum groups at roots of unity. Our treatment here, however, is self-contained and does not appeal to the general theory of Poisson Lie groups. Details on this subject can be found in [Dr1, STS, KS-Ma, Lu-We, Lu2].

In $\S 2$, we recall the convexity theorems of Kostant, and in $\S 3$, we recall some basic facts from Poisson and symplectic geometry. The main result is presented in $\S 4$, where we define a Poisson structure on the subgroup $A N$ when $G$ is complex semisimple, and we show that it has the required properties. As a consequence, we obtain the nonlinear convexity theorem of Kostant. In $\S 5$, we make some remarks on the Poisson structure $\pi$ in relation with its linearization and quantum groups, as well as some future questions. In $\S 6$, we show how to obtain the nonlinear theorem for real flag manifolds by the same argument as Duistermaat [Du1] used in the linear case. This was pointed out to us by Alan Weinstein. 


\section{THE CONVEXITY THEOREM OF KOSTANT}

In this section, we review the convexity theorem of Kostant [Kt]. We first recall the Cartan and Iwasawa decompositions for a semisimple Lie group. See [He] for more details.

Let $G$ be a real connected semisimple Lie group and $\mathfrak{g}$ its Lie algebra. Let $\mathfrak{k}$ be a subalgebra of $\mathfrak{g}$ corresponding to a maximal compact subgroup of the adjoint group of $G$, and let $\mathfrak{p}$ be the orthogonal complement of $\mathfrak{k}$ in $\mathfrak{g}$ with respect to the Killing form $E$ of $\mathfrak{g}$. Then $\mathfrak{g}$ is the direct sum of $\mathfrak{k}$ and $\mathfrak{p}$ as vector spaces; the decomposition $\mathfrak{g}=\mathfrak{k} \oplus \mathfrak{p}$ is called the Cartan decomposition of $\mathfrak{g}$. Elements of $\mathfrak{p}$ are semisimple, i.e., the linear map $\operatorname{Ad}_{X}: \mathfrak{g} \rightarrow \mathfrak{g}$ is diagonalizable for $X \in \mathfrak{p}$. It follows that $P:=\exp \mathfrak{p}$ is a closed submanifold of $G$, diffeomorphic to $\mathfrak{p}$ via the exponential map. Let $K$ be the connected subgroup of $G$ with $\mathfrak{k}$ as its Lie algebra. The Cartan decomposition for $G$ says that $G=K P$, i.e., the map $K \times P \rightarrow G:(k, p) \mapsto k p$ is a diffeomorphism. By the invariance of the Killing form, we have $[\mathfrak{k}, \mathfrak{p}] \subset \mathfrak{p}$, so $K$ acts on $\mathfrak{p}$ by the adjoint action and on $P$ by conjugations, with the exponential map from $\mathfrak{p}$ to $P$ being $K$-equivariant.

Let $\mathfrak{a}$ be a maximal abelian subspace in $\mathfrak{p}$. Since $\mathfrak{a}$ consists of semisimple elements, the adjoint representation of $\mathfrak{a}$ on $\mathfrak{g}$ decomposes $\mathfrak{g}$ into the direct sum of the eigenspaces $\mathfrak{g}=\mathfrak{l} \oplus \sum_{\lambda \in \Delta} \mathfrak{g}_{\lambda}$, where $\mathfrak{l}$ is the centralizer of $\mathfrak{a}$ in $\mathfrak{g}$, and for a linear functional $\lambda$ on $\mathfrak{a}, \mathfrak{g}_{\lambda}$ denotes the set of all $X \in \mathfrak{g}$ such that $[H, X]=\lambda(H) X$ for all $H \in \mathfrak{a}$, and $\Delta$ is the set of all such nonzero $\lambda$ 's. Fixing a basis for $\mathfrak{a}$, we can then compare any two linear functionals on $\mathfrak{a}$ in the lexicographical way. Put $\mathfrak{n}=\sum_{\lambda>0} \mathfrak{g}_{\lambda}$. Then $\mathfrak{n}$ is a nilpotent subalgebra of $\mathfrak{g}$, and $\mathfrak{g}=\mathfrak{k} \oplus \mathfrak{a} \oplus \mathfrak{n}$ as vector spaces. Let $A$ and $N$ be respectively the connected subgroups of $G$ with $\mathfrak{a}$ and $\mathfrak{n}$ as Lie algebras. The theorem of Iwasawa states that the map $(k, a, n) \mapsto k a n, k \in K, a \in A, n \in N$, is a diffeomorphism of $K \times A \times N$ with $G$. The decomposition $G=K A N$ is called an Iwasawa decomposition of $G$. We will use $W$ to denote the (relative) Weyl group of $(K, \mathfrak{a})$; it is defined as the quotient of the normalizer of $\mathfrak{a}$ in $K$ by the centralizer of $\mathfrak{a}$ in $K$.

If $G$ is a complex semisimple Lie group but considered as a real Lie group, we can choose $\mathfrak{k}$ to be a compact real form of $\mathfrak{g}$. The Killing form of $\mathfrak{g}$ is the complex linear extension of that of $\mathfrak{k}$ to $\mathfrak{g}$. Therefore we have $\mathfrak{p}=i \mathfrak{k}$. If $\mathfrak{t}$ is a Cartan subalgebra of $\mathfrak{k}$, then $\mathfrak{a}=i$ it a maximal abelian subspace of $\mathfrak{p}$, and the Weyl group of $(K, \mathfrak{t})$ is the same as that of $(K, \mathfrak{a})$. Any choice of a basis for $\mathfrak{t}$ will define an Iwasawa decomposition for $\mathfrak{g}$ and $G$.

Example 2.1. Let $G=\mathrm{SL}(n, \mathbb{C}), K=\mathrm{SU}(n), P$ the space of all $n \times n$ positive definite Hermitian matrices of determinant $1, A$ the group of all $n \times n$ positive definite diagonal matrices with determinant 1 , and finally $N$ the group of all $n \times n$ strictly upper triangular (complex) matrices. Then $\mathrm{SL}(n, \mathbb{C})=\mathrm{SU}(n) P$ is a Cartan decompositionand $\mathrm{SL}(n, \mathbb{C})=\mathrm{SU}(n) A N$ is an Iwasawa decompo- 
sition of $\operatorname{SL}(n, \mathbb{C})$. Similarly, replacing $\mathrm{SU}(n)$ by $\mathrm{SO}(n)$, and "complex" by "real," we get a Cartan and an Iwasawa decomposition of $\operatorname{SL}(n, \mathbb{R})$.

The two decompositions $G=K P$ and $G=K A N$ give rise to an identification between the space $P$ and the group $A N$; namely, if $p \in P$ is written as $p=k a n$ for some $k \in K, a \in A$ and $n \in N$, then $p$ is identified with $a n \in A N$. We will use $\rho_{A}$ to denote the projection from $P$ (or $G$, or $A N$ ) to $A: \rho_{A}(p=k a n)=a$. Kostant's nonlinear convexity theorem concerns the image of a $K$-orbit in $P$ under the projection $\rho_{A}$.

Theorem 2.2. (Kostant $[\mathrm{Kt}]$ ). Let $G$ be a connected semisimple Lie group, and let $G=K P$ and $G=K A N$ with, $A \subset P$, be respectively a Cartan and an Iwasawa decomposition of $G$. For $a \in A$, let $\mathscr{O}_{a}$ be the $K$-orbit of $a$ in $P$. Then the image of $\mathscr{O}_{a}$ under the projection $\rho_{A}$ is the convex hull of the Weyl group orbit $W \cdot a$ in $A$. Here, we identify $A$ with its Lie algebra $\mathfrak{a}$ by the exponential map; thus convexity in A makes sense.

To justify the term "nonlinear," we recall the linear convexity theorem. The linearization of $\rho_{A}: P \rightarrow A$ at the identity element $e \in G$ gives rise to the orthogonal projection $\rho_{\mathfrak{a}}$ from $\mathfrak{p}$ to $\mathfrak{a}$ with respect to the Killing form of $\mathfrak{g}$. We compare $\rho_{\mathfrak{a}}$ with the map $J_{1}:=\log \circ \rho_{A} \circ \exp$, where $\log : A \rightarrow \mathfrak{a}$ is the inverse of the exponential map of $A$. Both of them are from $\mathfrak{p}$ to $\mathfrak{a}$. With respect to the linear space structures of $\mathfrak{p}$ and $\mathfrak{a}, \rho_{\mathfrak{a}}$ is linear, while $J_{1}$ is highly nonlinear as seen from the example below. But the linearization of $J_{1}$ at the zero point of $\mathfrak{p}$ is just $\rho_{a}$. Now the full convexity theorem of Kostant states that each $K$-orbit $\mathscr{O}_{X}$ in $\mathfrak{p}$ has the same convex image under both the nonlinear map $J_{1}$ and its linearization $\rho_{\mathfrak{a}}$; this convex set is in fact the convex hull of the Weyl orbit $W \cdot X$.

Example 2.3. Take $\mathfrak{g}=\operatorname{sl}(n, \mathbb{C}), \mathfrak{k}=\operatorname{su}(n), \mathfrak{a}=$ the space of all real diagonal traceless matrices, and $\mathfrak{p}=$ the space of all traceless Hermitian matrices. Then for $X \in \mathfrak{p}, \rho_{\mathfrak{a}}$ simply picks up the diagonal part of $X$, while

$$
\begin{aligned}
J_{1}(X) & =\left(\log \circ \rho_{A} \circ \exp \right)(X) \\
& =\frac{1}{2}\left(\log \Delta_{1}\left(e^{2 X}\right), \log \frac{\Delta_{2}\left(e^{2 X}\right)}{\Delta_{1}\left(e^{2 X}\right)}, \ldots, \log \frac{\Delta_{n}\left(e^{2 X}\right)}{\Delta_{n-1}\left(e^{2 X}\right)}\right),
\end{aligned}
$$

where for a matrix $S=\left(s_{i j}\right)_{i, j=1, \ldots n}, \Delta_{k}(S)$ denotes the determinant of the submatrix $\left(s_{i j}\right)_{i, j=1, \ldots, k}$. It is clear that $J_{1}$ is highly nonlinear. For a given $X=\left(x_{1}, \ldots, x_{n}\right) \in \mathfrak{a}$, the flag manifold $\mathscr{O}_{X}$ is the set of all Hermitian matrices with $\left\{x_{1}, \ldots, x_{n}\right\}$ as their eigenvalues. The relative Weyl group $W$ is just the permutation group $\Sigma_{n}$ of $n$ elements. Therefore, the linear convexity theorem of Kostant restates a known result of Schur and Horn [Ho] [Sc].

The linear convexity theorem of Kostant has been shown to fit into symplectic geometry by Atiyah [Ay], Duistermaat [Du1], and Guillemin-Sternberg [Gu-St] by realizing that (1) in the case of complex flag manifolds the projection $\rho_{\mathfrak{a}}$ 
is a moment map for a Hamiltonian torus action and (2) a real flag manifold could be thought of as the fixed point set of an anti-symplectic involution on a complex flag manifold. Our approach for the nonlinear theorem follows the same line.

\section{POISSON AND SYMPLECTIC GEOMETRY}

A Poisson structure on a manifold $M$ [We] is a bivector field $\pi$ on $M$ such that the bracket operation on the algebra $C^{\infty}(M)$ of smooth functions on $M$ defined by

$$
\{\phi, \varphi\}=\pi(d \phi, d \varphi), \quad \phi, \varphi \in C^{\infty}(M),
$$

satisfies Jacobi's identity. $\{\phi, \varphi\}$ is called the Poisson bracket of $\phi$ and $\varphi$. Purely in terms of the bivector field $\pi$, the Jacobi identity of the Poisson bracket can be expressed as $[\pi, \pi]=0$, where $[$,$] denotes the Schouten bracket on$ the space multivector fields on $M([\mathrm{Ku}])$.

The bivector field $\pi$ can also be regarded as the bundle map

$$
\pi^{\#}: T^{*} M \longrightarrow T M: \beta\left(\pi^{\#}(\alpha)\right)=\pi(\beta, \alpha) .
$$

For a smooth function $f$ on $M$, the vector field $\pi^{\#}(d f)$ is called the Hamiltonian vector field of $f$. When $\pi$ is of maximal rank ( $M$ is then necessarily even dimensional), the bundle map $\left(\pi^{\#}\right)^{-1}: T M \rightarrow T^{*} M$ defines a nondegenerate closed 2-form $\omega$ on $M$ by $i_{X} \omega=\left(\pi^{\#}\right)^{-1}(X)$, where $X$ is a vector field on $M$ and $i$ denotes the interior product of vector fields and differential forms. A manifold with a nondegenerate closed 2-form is called a symplectic manifold. In general, $\pi$ may have varying (but necessarily even) ranks. The image of the bundle map $\pi^{\#}: T^{*} M \rightarrow T M$ defines a singular involutive distribution on $M$. Integral submanifolds of this distribution naturally inherit symplectic structures, and they are called the symplectic leaves of $(M, \pi)$. Therefore, symplectic manifolds are special cases of Poisson manifolds and every Poisson manifold is foliated by symplectic manifolds.

Obvious symmetries of a Poisson manifold $(M, \pi)$ are described by Lie group actions on $M$ leaving $\pi$ invariant. Let $\sigma: G \times M \rightarrow M$ be such an action, and let $\mathfrak{g} \rightarrow \operatorname{Vect}(M): X \mapsto \sigma_{X}$ be the corresponding infinitesimal action of $\mathfrak{g}$ on $M$. The action is said to be Hamiltonian if there is a linear map $\mathfrak{g} \rightarrow$ $C^{\infty}(M): X \mapsto J_{X}$ such that (1) $\pi^{\#}\left(d J_{X}\right)=\sigma_{X}$, and (2) $J_{[X, Y]}=\left\{J_{X}, J_{Y}\right\}$, where $X, Y \in \mathfrak{g}$. In this case, the map $J: M \rightarrow \mathfrak{g}^{*}:(J(m), X)=J_{X}(m), m \in$ $M, X \in \mathfrak{g}$, is called a moment map for the action $\sigma$.

The convexity theorem of Atiyah-Guillemin-Sternberg is now stated as follows.

Theorem 3.1 (Atiyah [Ay]-Guillemin-Sternberg [Gu-St]). If $J: P \rightarrow \mathbb{R}^{k}$ is a moment map for a Hamiltonian torus $T^{k}$ action on a compact symplectic manifold $P$, then the image $J(P)$ is the convex hull of $J\left(P^{T^{k}}\right)$, where $P^{T^{k}}$ denotes the fixed point set of the action. 
We will use the following theorem of Duistermaat to study the case of real flag manifolds.

Theorem 3.2 (Duistermaat [Du1]). If $J: P \rightarrow \mathbb{R}^{k}$ is a moment map for a Hamiltonian torus $T^{k}$ action on a compact symplectic manifold $P$ and if $Q$ is the fixed point set (or a connected component of it) of an anti-symplectic involution of $P$ leaving $J$ invariant, then $J(Q)=J(P)$ and it is the convex hull of $J\left(P^{T^{k}} \cap Q\right)$.

\section{A Poisson structure on the Group $A N$}

In this section, we assume that $G$ is a complex semisimple Lie group regarded as a real Lie group. Let $K$ be the compact real form of $G$, and let $G=K A N$ be an Iwasawa decomposition of $G$. We will define a Poisson structure on the subgroup $A N$.

Notation 4.1. Let $\mathfrak{g}, \mathfrak{k}, \mathfrak{a}$, and $\mathfrak{n}$ be respectively the Lie algebras of $G, K, A$, and $N$. From now on, we will use $\mathfrak{b}$ to denote $\mathfrak{a} \oplus \mathfrak{n}$ and $B$ to denote $A N$. The Iwasawa decompositions are then $\mathfrak{g}=\mathfrak{k} \oplus \mathfrak{b}$ and $G=K B$. Elements of $\mathfrak{k}$ and $\mathfrak{b}$ will be denoted respectively by $X, Y, \ldots$ and $\xi, \eta, \ldots$ We will also denote the two projections from $\mathfrak{g}$ to its two factors $\mathfrak{k}$ and $\mathfrak{b}$ by $\rho_{\mathfrak{k}}$ and $\rho_{\mathfrak{b}}$ :

$$
\begin{gathered}
\rho_{\mathfrak{k}}: \mathfrak{g}=\mathfrak{k} \oplus \mathfrak{b} \longrightarrow \mathfrak{k}: X+\xi \longmapsto X, \\
\rho_{\mathfrak{b}}: \mathfrak{g}=\mathfrak{k} \oplus \mathfrak{b} \longrightarrow \mathfrak{b}: X+\xi \longmapsto \xi .
\end{gathered}
$$

The adjoint action of $G$ on $\mathfrak{g}$ will be denoted by Ad. For $b \in B$ and $X \in \mathfrak{k}$, the element $\operatorname{Ad}_{b} X \in \mathfrak{g}$ will, in general, have both $\mathfrak{k}$ and $\mathfrak{b}$ components.

Let $E$ be the (complex-valued) Killing form $E$ of $\mathfrak{g}$ as a complex Lie algebra, and let $\langle$,$\rangle be its imaginary part. Then \langle$,$\rangle is symmetric, nondegener-$ ate, and invariant. Since $\mathfrak{k}$ is a real form of $\mathfrak{g}$, we know that $\langle\mathfrak{k}, \mathfrak{k}\rangle=0$. It also follows from the fact that $E\left(\mathfrak{g}_{\lambda}, \mathfrak{g}_{\mu}\right)=0$ for $\lambda+\mu \neq 0$ that $\langle\mathfrak{a} \oplus \mathfrak{n}, \mathfrak{a} \oplus \mathfrak{n}\rangle=0$. Therefore, using $\langle$,$\rangle , we can identify \mathfrak{k}$ with the dual space of $\mathfrak{b}$. We are now ready to define the Poisson structure on $B=A N$.

Definition 4.2. On the subgroup $B=A N$, define a bivector field $\pi$ by

$$
\left(r_{b^{-1}} \pi(b)\right)(X, Y)=\left\langle\rho_{\mathfrak{k}}\left(\operatorname{Ad}_{b^{-1}} X\right), \rho_{\mathfrak{b}}\left(\operatorname{Ad}_{b^{-1}} Y\right)\right\rangle \text {, }
$$

where $X, Y \in \mathfrak{k}$ are considered as elements of the dual space $\mathfrak{b}^{*}$ of $\mathfrak{b}$ via $\langle$,$\rangle , and for b \in B, r_{b}$ denotes both the right translation in $B$ by $b$, as well as its differential map extended to bivector fields on $B$.

We need to show that $\pi$ is a Poisson structure on $B=A N$.

Lemma 4.3. $\pi$ is skew-symmetric.

Proof. Since $\langle$,$\rangle is invariant, we have for any X, Y \in \mathfrak{k}$ and $b \in B$, that

$$
\begin{aligned}
0 & =\langle X, Y\rangle=\left\langle\operatorname{Ad}_{b^{-1}} X, \operatorname{Ad}_{b^{-1}} Y\right\rangle \\
& =\left\langle\rho_{\mathfrak{k}}\left(\operatorname{Ad}_{b^{-1}} X\right), \rho_{\mathfrak{b}}\left(\operatorname{Ad}_{b^{-1}} Y\right)\right\rangle+\left\langle\rho_{\mathfrak{b}}\left(\operatorname{Ad}_{b^{-1}} X\right), \rho_{\mathfrak{k}}\left(\operatorname{Ad}_{b^{-1}} Y\right)\right\rangle .
\end{aligned}
$$

Hence $\pi$ is skew-symmetric. 
The next lemma shows that $\pi$ is compatible with the group structure of $B=$ $A N$ in the sense that the group multiplication map $B \times B \rightarrow B:\left(b_{1}, b_{2}\right) \mapsto b_{1} b_{2}$ is a Poisson map; $B \times B$ is equipped with the direct product Poisson structure $\pi \oplus \pi$.

Lemma 4.4. For $b_{1}, b_{2} \in B$, we have that

$$
\pi\left(b_{1} b_{2}\right)=l_{b_{1}} \pi\left(b_{2}\right)+r_{b_{2}} \pi\left(b_{1}\right) .
$$

Proof. Set $\pi_{r}(b)=r_{b^{-1}} \pi(b)$ for $b \in B$. Equation (1) then becomes the following cocycle condition:

$$
\pi_{r}\left(b_{1} b_{2}\right)=\pi_{r}\left(b_{1}\right)+\operatorname{Ad}_{b_{1}} \pi_{r}\left(b_{2}\right), \quad \forall b_{1}, b_{2} \in B .
$$

Let $X, Y \in \mathfrak{k} \cong \mathfrak{b}^{*}$. Then,

$$
\begin{aligned}
\pi_{r}\left(b_{1} b_{2}\right)(X, Y)= & \left\langle\rho_{\mathfrak{k}} \operatorname{Ad}_{\left(b_{1} b_{2}\right)^{-1}} X, \rho_{\mathfrak{b}} \operatorname{Ad}_{\left(b_{1} b_{2}\right)^{-1}} Y\right\rangle \\
= & \left\langle\operatorname{Ad}_{b_{1}^{-1}} X, \operatorname{Ad}_{b_{2}} \rho_{\mathfrak{b}} \operatorname{Ad}_{\left(b_{1} b_{2}\right)^{-1}} Y\right\rangle \\
= & \left\langle\rho_{\mathfrak{k}} \operatorname{Ad}_{b_{1}^{-1}} X, \operatorname{Ad}_{b_{2}} \rho_{\mathfrak{b}} \operatorname{Ad}_{b_{2}^{-1}}\left(\operatorname{Ad}_{b_{1}^{-1}} Y\right)\right\rangle \\
= & \left\langle\rho_{\mathfrak{k}} \operatorname{Ad}_{b_{1}^{-1}} X, \operatorname{Ad}_{b_{2}} \rho_{\mathfrak{b}} \operatorname{Ad}_{b_{2}^{-1}} \rho_{\mathfrak{b}} \operatorname{Ad}_{b_{1}^{-1}} Y\right\rangle \\
& +\left\langle\rho_{\mathfrak{k}} \operatorname{Ad}_{b_{1}^{-1}} X, \operatorname{Ad}_{b_{2}} \rho_{\mathfrak{b}} \operatorname{Ad}_{b_{2}^{-1}} \rho_{\mathfrak{k}} \operatorname{Ad}_{b_{1}^{-1}} Y\right\rangle \\
= & \pi_{r}\left(b_{1}\right)(X, Y)+\pi_{r}\left(b_{2}\right)\left(\rho_{\mathfrak{k}} \operatorname{Ad}_{b_{1}^{-1}} X, \rho_{\mathfrak{k}} \operatorname{Ad}_{b_{1}^{-1}} Y\right) .
\end{aligned}
$$

Notice that $\rho_{\mathfrak{k}} \operatorname{Ad}_{b_{1}^{-1}} X \in \mathfrak{k} \cong \mathfrak{b}^{*}$ is just the coadjoint action of $b \in B$ on $X \in$ $\mathfrak{b}^{*}$. Hence the last term in the above equation becomes $\left.\operatorname{(Ad}_{b_{1}} \pi_{r}\left(b_{2}\right)\right)(X, Y)$. This proves the cocycle condition (2).

Definition 4.5. We say that a multivector field $\Pi$ on a Lie group $B$ is multiplicative if

$$
\Pi\left(b_{1} b_{2}\right)=l_{b_{1}} \Pi\left(b_{2}\right)+r_{b_{2}} \Pi\left(b_{1}\right)
$$

for all $b_{1}, b_{2} \in B$.

Lemma 4.6. Assume that $B$ is connected. Then,

(1) a multivector field $\Pi$ is multiplicative if and only if $\Pi(e)=0$ and $\mathscr{L}_{V} \Pi$ is left invariant whenever $V$ is a left invariant (one)-vector field on $B$, where $e \in B$ is the identity element of $B$ and $\mathscr{L}_{V} \Pi$ denotes the Lie derivative of $\Pi$ in the direction of $V$;

(2) the Schouten bracket of two multiplicative multivector fields is again multiplicative;

(3) a multiplicative multivector field is identically zero on $B$ if and only if its derivative at $e$ is zero.

Proof. By letting $b_{1}=b_{2}=e$, we see that a necessary condition for $\Pi$ to be multiplicative is that $\Pi(e)=0$. Replacing $b_{2}$ by $\exp t V$ in (3) and differentiating with respect to $t$ gives rise to the left invariant of $\mathscr{L}_{V} \Pi$. Conversely, the left invariance of $\mathscr{L}_{V} \Pi$ for every left invariant $V$ implies that (3) holds 
for any $b_{1} \in B$ and $b_{2}=\exp t V$. Since $B$ is connected, it is generated by any open neighborhood of $e$. It follows that (3) holds for all $b_{1}, b_{2} \in B$. Assume now that $\Pi_{1}$ and $\Pi_{2}$ are two multiplicative multivector fields on $B$. Let $V$ be any left invariant vector field on $B$. We have that

$$
\mathscr{L}_{V}\left[\Pi_{1}, \Pi_{2}\right]=\left[\mathscr{L}_{V} \Pi_{1}, \Pi_{2}\right]+\left[\Pi_{1}, \mathscr{L}_{V} \Pi_{2}\right]
$$

By (1) and by the product rule of the Schouten bracket, the right-hand side of the above equation is left invariant. Again by $(1),\left[\Pi_{1}, \Pi_{2}\right]$ is multiplicative. This proves (2). Now if $\Pi$ is multiplicative, then $\Pi(e)$ is necessarily zero. We can then take the (intrinsic) derivative of $\Pi$ at $e$. It is a linear map $d_{e} \Pi$ from $\mathfrak{b}$ to $\Lambda^{l} \mathfrak{b}$, where $l$ is the degree of $\Pi$, defined by $d_{e} \Pi(\xi)=\left(\mathscr{L}_{V} \Pi\right)(e)$, where $V$ can be any vector field on $B$ with value $\xi$ at $e$. In particular, $V$ can be the left invariant vector field defined by $\xi$. Therefore if $d_{e} \Pi=0$, then by (1) $\mathscr{L}_{V} \Pi=0$ for all left invariant vector field $V$ on $B$. Since left invariant vector fields generate right translations, we know that $\Pi$ is right invariant. But $\Pi(e)=0$, and thus $\Pi=0$.

Now let $\pi$ be a multiplicative bivector field on $B$. The dual map of $d_{e} \pi$ defines a skew-symmetric operation $[,]_{\pi}$ on $\mathfrak{b}^{*}$ by $\left(\xi,[X, Y]_{\pi}\right)=\left(d_{e} \pi(e)\right)(\xi) \times$ $(X, Y)$ for $\xi \in \mathfrak{b}$ and $X, Y \in \mathfrak{b}^{*}$.

Lemma 4.7. On a connected Lie group $B$, a multiplicative bivector field $\pi$ defines a Poisson structure if and only if its derivative at e defines a Lie algebra structure on $\mathfrak{b}^{*}$.

Proof. By definition, $\pi$ is Poisson if and only if $[\pi, \pi]$, the Schouten bracket of $\pi$ with itself, is zero. By Lemma 4.6, $[\pi, \pi]$ is also multiplicative, and it is zero if and only if its derivative $d_{e}[\pi, \pi]$ is zero. By using the definition of the Schouten bracket $[\mathrm{Ku}]$, one calculates (see also [Da-So]) that

$$
\left(d_{e}[\pi, \pi]\right)(X, Y, Z)=2\left[X,[Y, Z]_{\pi}\right]_{\pi}+2\left[Y,[Z, X]_{\pi}\right]_{\pi}+2\left[Z,[X, Y]_{\pi}\right]_{\pi}
$$

for $X, Y, Z \in \mathfrak{b}^{*}$. Therefore, $d_{e}[\pi, \pi]=0$ is equivalent to the Jacobi identity for $[,]_{\pi}$. This proves the lemma.

We now return to the bivector field $\pi$ on $B=A N$ defined in Definition 4.2.

Proposition 4.8. The dual map of the intrinsic derivative $d_{e} \pi$ of $\pi$ at $e$ is exactly the Lie bracket map on $\mathfrak{k}: \mathfrak{k} \wedge \mathfrak{k} \rightarrow \mathfrak{k}$. Here recall that $\mathfrak{k}$ is identified with the dual space of $\mathfrak{b}$ via the scalar product $\langle$,$\rangle on \mathfrak{g}$.

Proof. Let $\xi \in \mathfrak{b}$ and $X, Y \in \mathfrak{k}$. By definition, the pairingof $\xi$ and $[X, Y]_{\pi}$ is given by

$$
\begin{aligned}
\left(\xi,[X, Y]_{\pi}\right) & =\left.\frac{d}{d t}\right|_{t=0} r_{\exp (-t \xi)} \pi(\exp t \xi)(X, Y) \\
& =\left.\frac{d}{d t}\right|_{t=0}\left\langle\rho_{\mathfrak{k}} \operatorname{Ad}_{\exp (-t \xi)} X, \rho_{\mathfrak{b}} \operatorname{Ad}_{\exp (-t \xi)} Y\right\rangle \\
& =\left\langle X, \rho_{\mathfrak{b}}[-\xi, Y]\right\rangle=\langle X,-[\xi, Y]\rangle=\langle\xi,[X, Y]\rangle .
\end{aligned}
$$

Therefore $[X, Y]_{\pi}=[X, Y]$, the Lie bracket of $X$ and $Y$ in $\mathfrak{k}$. 
We have therefore proved the following theorem [Lu-We, Lu2].

Theorem 4.9. The bivector field $\pi$ as given in Definition 4.2 defines a Poisson structure on $B=A N$.

Remark 4.10. A Lie group together with a multiplicative Poisson structure is called a Poisson Lie group [Dr1, STS, Lu-We]. We have in fact showed that $B$ together with $\pi$ defined above is a Poisson Lie group.

We now turn to the study of the symplectic leaves of $\pi$. Recall that the Iwasawa decomposition states that $G=K B$. We will use $\rho_{B}: G \rightarrow B$ to denote the projection from $G$ to $B$ with respect to this decomposition. Then an action of $K$ on $B$ can be defined as

$$
\sigma: K \times B \longrightarrow B:(k, b) \longmapsto \rho_{B}\left(k b k^{-1}\right), \quad k \in K, b \in B .
$$

This is indeed an action. If we identify $B$ with the coset space $K \backslash G$, then it is just the right action of $K$ on $K \backslash G$ by right translations made into a left one by replacing $k$ by $k^{-1}$. For $X \in \mathfrak{k}$, the infinitesimal generator of the action in the direction $X$ is given by $\sigma_{X}(b)=-r_{b} \rho_{b}\left(\operatorname{Ad}_{b} X\right)$ for $b \in B$. On the other hand, each $X \in \mathfrak{k}$ can be considered as a covector at $e \in B$, so it defines a left invariant 1 -form $X^{l}$ on $B$. Let $\pi^{\#}: T^{*} B \rightarrow T B$ be the bundle map defined by $\pi$. The symplectic leaves of $\pi$ are by definition the integral manifolds of the distribution in $B$ defined by the image of this bundle map.

Theorem 4.11. For any $X \in \mathfrak{k}$, we have $\pi^{\#}\left(X^{l}\right)=\sigma_{X}$. Therefore the symplectic leaves of $\pi$ in $B$ are exactly the orbits of the $K$-action $\sigma$ on $B$.

Proof. Let $Y \in \mathfrak{k} \cong \mathfrak{b}^{*}$, and let $Y^{r}$ be the right invariant 1 -form on $B$ defined by $Y$. Then the pairing of $Y^{r}$ with $\pi^{\#}\left(X^{l}\right)$ at a point $b \in B$ is given by

$$
\begin{aligned}
\left(Y^{r}, \pi^{\#}\left(X^{l}\right)\right)(b) & =\pi(b)\left(Y^{r}, \rho_{\mathfrak{k}} \operatorname{Ad}_{b} X\right) \\
& =\left\langle\rho_{\mathfrak{k}} \operatorname{Ad}_{b^{-1}} Y, \rho_{\mathfrak{b}} \operatorname{Ad}_{b^{-1}} \rho_{\mathfrak{k}} \operatorname{Ad}_{b} X\right\rangle \\
& =\left\langle\rho_{\mathfrak{k}} \operatorname{Ad}_{b^{-1}} Y,-\rho_{\mathfrak{b}} \operatorname{Ad}_{b^{-1}} \rho_{\mathfrak{b}} \operatorname{Ad}_{b} X\right\rangle \\
& =-\left\langle Y, \rho_{\mathfrak{b}} \operatorname{Ad}_{b} X\right\rangle=\left(Y^{r}, \sigma_{X}\right)(b) .
\end{aligned}
$$

Therefore $\pi^{\#}\left(X^{l}\right)=\sigma_{X}$ for all $X \in \mathfrak{k}$.

Remark 4.12. Theorem 4.11 can be thought of as another description of $\pi$, namely, regarded as a bundle map from the cotangent bundle $T^{*} B$ to the tangent bundle $T B, \pi$ is the unique one that maps each left invariant 1 -form $X^{l}$ to the vector field $\sigma_{X}$ for $X \in \mathfrak{k}$.

Let $T$ be the connected subgroup of $K$ with Lie algebra $\mathfrak{t}=i \mathfrak{a}$. It is a maximal torus of $K$.

Theorem 4.13. The restriction of $\sigma$ to $T$ leaves the Poisson structure $\pi$ on $B$ invariant and the map

$$
J:=\log \circ \rho_{A}: B=A N \longrightarrow \mathfrak{a}: \text { an } \longmapsto \log (a)
$$

is a moment map for this $T$-action. 
Proof. Since $T$ commutes with $A$ and since $N$ is a normal subgroup of $T A N$, we know that $J$ is invariant under $T$. For $X \in \mathfrak{t}$, let $J_{X} \in C^{\infty}(A N)$ be the $X$-component of $J$, i.e., $J_{X}(b)=\langle J(b), X\rangle$ for $b \in A N$. Then the differential $d J_{X}$ is exactly the left invariant 1 -form on $A N$ with value $X$ at the identity. Therefore by Theorem 4.11, $\pi^{\#}\left(d J_{X}\right)=\sigma_{X}$. This, together with the $T$-invariance of $J$, implies that $J$ is an equivariant moment map for the $T$-action.

Remark 4.14. The Poisson structure $\pi$ on $B$ is not invariant under the whole $K$-action. Namely, each individual element $k \in K$ does not necessarily preserve $\pi$, unless $k \in T$. In fact there is a nonzero Poisson structure defined on $K$ such that the action map $K \times B \rightarrow B$ is a Poisson map, where $K \times B$ has the direct product Poisson structure. See [Lu-We]. This is an example of a Poisson action by a Poisson Lie group. There is a similar theory of moment maps and reductions for such actions [Lu1]. When the Poisson structure on the Lie group is zero, this reduces to the corresponding theory in the usual case. The Poisson structure on $K$ mentioned above vanishes on $T$. This is why we have a Hamiltonian torus action in the usual sense.

Proof of Kostant's Theorem (Theorem 2.2) in the complex case. We now identify $B=A N$ with the symmetric space $P=\exp i$ p by identifying both of them with the quotient space $K \backslash G$, or more precisely by $P \ni p \mapsto a n \in B$ if $p=k a n$ for $k \in K, a \in A$ and $n \in N$. The action $\sigma$ of $K$ on $B$ then becomes the action of $K$ on $P$ by conjugation. We still use $\pi$ to denote the induced Poisson structure on $P$ by this identification. Then by Theorem 4.11, the symplectic leaves of $\pi$ are exactly the orbits of the $K$ action on $P$. By Theorem 4.13, $T$ leaves $\pi$ invariant and it has the map $P \rightarrow \mathfrak{a}: p=k a n \mapsto \log a$ as an equivariant moment map. It is well-known that every $K$-orbit in $P$ intersects with $A$, and the fixed point set of the $T$-action on the $K$-orbit $\mathscr{O}_{a}$ through $a \in A$ is exactly the Weyl group orbit $W \cdot a$. Applying Theorem 3.1, we obtain the nonlinear convexity theorem for complex flag manifolds (Theorem 2.2).

\section{SOME REMARKS}

In this section, we make some remarks on the Poisson structure $\pi$ defined in $\S 4$.

Linearization of $\pi$. Recall that $P$ can be identified with the linear space $\mathfrak{p}=i \mathfrak{k}$ via the exponential map. Correspondingly, we can think of $\pi$ as defined on $\mathfrak{p}$. Its symplectic leaves are again $K$-orbits, but this time $K$ acts on $\mathfrak{p}$ by the adjoint action. On the other hand, we can also identify $\mathfrak{p}$ with the dual space of $\mathfrak{k}$ (via the imaginary part of the (complex-valued) Killing form of $\mathfrak{g}$, namely the scalar product $\langle$,$\rangle in \S 4$ ). So there is also a Lie Poisson structure $\pi_{0}$ on $\mathfrak{p}$ [We] that has the same symplectic leaves as $\pi$ does. Moreover, $\pi_{0}(0)=0$, and by Proposition 4.8, the linearization of $\pi$ at 0 is just $\pi_{0}$. Since $\mathfrak{k}$ is a compact semisimple Lie algebra, a theorem of Conn [Co] says that locally around the zero point of $\mathfrak{p}, \pi$ is diffeomorphic to $\pi_{0}$. 
Question 5.1. Are $\pi$ and $\pi_{0}$ globally diffeomorphic as Poisson structures?

Such a diffeomorphism, if exists, cannot be $K$-equivariant because $\pi_{0}$ is $K$ invariant while $\pi$ is not. But we strongly suspect that a $T$-equivariant Poisson diffeomorphism exists. Recently, Ping Xu [Xu] has given such an explicit diffeomorphism for the case of $K=\mathrm{SU}(2)$. On the other hand, by looking at the family of Poisson structures on $\mathfrak{p}$ defined by $\pi_{h}(X)=\frac{1}{h} \pi(h X)$ for $h \neq 0$ and their moment maps for the $T$-action, Viktor Ginzburg [Gz] has observed that their induced symplectic structures on each leaf have the same cohomology class and therefore are diffeomorphic as symplectic structures. We would also like to call the reader's attention to [Du2], where the moment maps for $\pi$ and $\pi_{0}$ are shown to be diffeomorphic by a homotopy argument. We thank Professor Hermann Flaschka for pointing the reference [Du2] to us.

Relation with quantum groups [Wo, Dr2, Co-Kc, C-K-P]. The Poisson structure on $K$ that we mentioned in Remark 4.14 makes $K$ into a Poisson Lie group [STS], which can be regarded as the semiclassical limit of the quantum group corresponding to $K$, and the group $B=A N$ with the Poisson structure $\pi$ is the dual Poisson Lie group of $K$. Moreover the $K$-action $\sigma$ on $B$ defined by Formula (4) is the so-called dressing action, which generalizes the coadjoint action to the case of Poisson Lie groups. Therefore, keeping in mind the orbit method in the representation theory of Lie groups, it is natural to ask how the "quantization" of the symplectic structure on each $K$-orbit in $B$ is related to the representations of the corresponding quantum group. Indeed it has been observed by Reshetikhin [Rs] and de Concini, Kac, and Procesi [Co-Kc, C-KP] that the Poisson structure $\pi$ can be used to define the so-called "quantized coadjoint action" for quantum groups at root of unity. But since $\pi$ is not $K$-invariant, the already existing geometric quantization theory does not apply. This leads to the following question:

Question 5.2. How to geometrically quantize the (K-noninvariant) symplectic structure on each $K$-orbit in $B$ (or in $\mathfrak{p})$ ?

\section{THE CASE OF REAL FLAG MANIFOLDS}

In $\S 4$, we assumed that $G$ was a complex semisimple Lie group but regarded as a real one. If $G$ is a real form of a complex Lie group, such as $G=\operatorname{SL}(n, \mathbb{R})$, the $K$-orbits in $P$ (called real flag manifolds) can have odd dimensions, so they do not admit symplectic structures. However, a real flag manifold should be considered as the fixed point set of an anti-symplectic involution of a complex flag manifold. This is the idea that Duistermaat used to put the linear convexity theorem into the symplectic framework. We show in this section that the same approach works for the nonlinear convexity theorem.

Let $G$ be a connected semisimple Lie group over $\mathbb{R}$ with Lie algebra $\mathfrak{g}$. Without loss of generality, we assume that $G$ has trivial center so that it admits a complexification $G_{1}$. The Lie algebra $\mathfrak{g}_{1}$ of $G_{1}$ is then the complexification 
of $\mathfrak{g}$, i.e., $\mathfrak{g}_{1}=\mathfrak{g}+i \mathfrak{g}$. We use $\tau$ to denote the conjugation of $\mathfrak{g}_{1}$ defined by $\mathfrak{g}$, namely $\tau(X+i Y)=X-i Y$ for $X, Y \in \mathfrak{g}$. It is an automorphism of $\mathfrak{g}_{1}$. There is also a unique automorphism of $G_{1}$, also denoted by $\tau$, whose differential at the identity element is equal to $\tau$. The connected identity component of the fixed point set of $\tau$ is $G$.

Let $\mathfrak{g}=\mathfrak{k} \oplus \mathfrak{p}$ be a Cartan decomposition of $\mathfrak{g}$. Define $\mathfrak{k}_{1}=\mathfrak{k}+i \mathfrak{p}$ and $\mathfrak{p}_{1}=i \mathfrak{k}+\mathfrak{p}=i \mathfrak{k}_{1}$. Then both $\mathfrak{k}_{1}$ and $\mathfrak{p}_{1}$ are invariant under $\tau$ and the fixed point set of $\left.\tau\right|_{\mathfrak{k}_{1}}$ and $\left.\tau\right|_{\mathfrak{p}_{1}}$ are respectively $\mathfrak{k}$ and $\mathfrak{p}$. Moreover, $\mathfrak{k}_{1}$ is a compact real form of $\mathfrak{g}_{1}$, and $\mathfrak{g}_{1}=\mathfrak{k}_{1} \oplus \mathfrak{p}_{1}$ is a Cartan decomposition of $\mathfrak{g}_{1}$. Let $\mathfrak{a}$ be a maximal abelian subspace of $\mathfrak{p}$, and let $\mathfrak{a}$ ' be any maximal abelian subalgebra of $\mathfrak{g}$ containing $\mathfrak{a}$. Define $\mathfrak{a}_{1}=\mathfrak{a}+i(\mathfrak{a} \cap \mathfrak{k})$. Then $\mathfrak{a}_{1}$ is a maximal abelian subspace of $\mathfrak{p}_{1}$, and $\mathfrak{a} \subset \mathfrak{a}_{1}$ is the fixed point set of $\tau$ in $\mathfrak{a}_{1}$. In defining lexicographic orderings on the dual spaces of $\mathfrak{a}$ and $\mathfrak{a}_{1}$, we choose a basis $X_{1}, \ldots, X_{n}$ of $\mathfrak{a}_{1}$ such that $X_{1}, \ldots, X_{m}$ is a basis for $\mathfrak{a}$, where $n=\operatorname{dim} \mathfrak{a}_{1}$ and $m=\operatorname{dim} \mathfrak{a}$. Then in the corresponding Iwasawa decompositions of $\mathfrak{g}$ and $\mathfrak{g}_{1}$ :

$$
\mathfrak{g}=\mathfrak{k} \oplus \mathfrak{a} \oplus \mathfrak{n}, \quad \mathfrak{g}_{1}=\mathfrak{k}_{1} \oplus \mathfrak{a}_{1} \oplus \mathfrak{n}_{1},
$$

$\mathfrak{n}_{1}$ is invariant under $\tau$ and $\mathfrak{n} \subset \mathfrak{n}_{1}$ is the fixed point set of $\tau$ in $\mathfrak{n}_{1}$.

On the group level, let $G=K P, G=K A N, G_{1}=K_{1} P_{1}$ and $G_{1}=K_{1} A_{1} P_{1}$ be respectively the Cartan and Iwasawa decompositions of $G$ and $G_{1}$. Then $K_{1}, P_{1}, N_{1}$, and $A_{1}$ are all invariant under $\tau$, and $K, P, A$, and $N$ are respectively (in the case of $K$, the identity component of) the fixed point set of $\tau$ in $K_{1}, P_{1}, A_{1}$, and $N_{1}$. Let $a \in A$, and let $\mathscr{O}_{a}$ (a real flag manifold) and $\mathscr{O}_{a, 1}$ (a complex flag manifold) be respectively the $K$ and $K_{1}$ orbits in $P$ and $P_{1}$ through $a$. Since $\tau(a)=a$ and since $K_{1}$ is $\tau$ invariant, we know that $\mathscr{O}_{a, 1}$ is $\tau$ invariant. The fixed point set of $\tau$ in $\mathscr{O}_{a, 1}$ may not be connected, but its $a$-connected component is $\mathscr{O}_{a}$.

Proposition 6.1. Let $\pi$ be the Poisson structure on $B_{1}=A_{1} N_{1}$ as defined in Definition 4.2. Then $\tau$ restricted to $B_{1}$ is anti-Poisson with respect to $\pi$, namely, $\tau_{*} \pi=-\pi$, where $\tau_{*}$ denotes the differential of $\tau$.

Proof. Lemma 4.4 says that $\pi$ is multiplicative on $B_{1}$. Since $\tau$ is a a group automorphism, $\tau_{*} \pi$, defined by $\tau_{*} \pi\left(b_{1}\right)=\tau_{*} \pi\left(\tau^{-1}\left(b_{1}\right)\right)$, is also multiplicative. By (3) of Lemma 4.6, it remains to show that the derivatives of $\tau_{*} \pi$ and $-\pi$ at $e$ are equal. We already know by Proposition 4.8 that the dual map of the derivative of $\pi$ at $e$ defines precisely the Lie algebra structure on $\mathfrak{k}_{1}$. Here recall that we are using the imaginary part of the Killing form $E$ of $\mathfrak{g}_{1}$, denoted by $\langle$,$\rangle in \S 4$, to identify $\mathfrak{k}_{1}$ with the dual space of $\mathfrak{b}_{1}=\mathfrak{a}_{1}+\mathfrak{n}_{1}$. Therefore, it suffices to show that the dual map of $\tau \mid \mathfrak{b}_{1}$, considered as defined on $\mathfrak{k}_{1}$, is a Lie algebra anti-automorphism of $\mathfrak{k}_{1}$. But the Killing form $E$ of $\mathfrak{g}_{1}$ is the complex linear extension to $\mathfrak{g}_{1}$ of the Killing form of $\mathfrak{g}$. It follows that for any $X$ and $\xi$ in $\mathfrak{g}_{1}$, we have $\langle X, \tau(\xi)\rangle=-\langle\tau(X), \xi\rangle$. In particular, by letting $X \in \mathfrak{k}_{1}$ and $\xi \in \mathfrak{b}_{1}$, we see that the dual map of $\left.\tau\right|_{\mathfrak{b}_{1}}$ becomes the map $-\tau$ 
on $\mathfrak{k}_{1}$ when we identify $\mathfrak{k}_{1}$ with $\mathfrak{b}_{1}^{*}$. Since $\tau$ is a Lie algebra automorphism on $\mathfrak{k}_{1},-\tau$ is a Lie algebra anti-automorphism. Therefore $\tau_{*} \pi=-\pi$, i.e., $\tau$ is anti-Poisson with respect to $\pi$.

Proof of Kostant's theorem (Theorem 2.2) in the real case. Identify the space $P_{1}$ with the group $B_{1}=A_{1} N_{1}$ by the map $p_{1}=k_{1} a_{1} n_{1} \mapsto a_{1} n_{1}$, and think of $\pi$ as defined on $P_{1}$. Since $\tau$ commutes with this identification map, it is again anti-Poisson with respect to $\pi$. Equipping each $K_{1}$-orbit $\mathscr{O}_{a, 1}$ in $P_{1}$ with the symplectic structure induced from $\pi, \tau$ becomes anti-symplectic. The $K$-orbit $\mathscr{O}_{a}$ is the $a$-component of the fixed point set of $\tau$ in $\mathscr{O}_{a, 1}$.

Let $T_{1}$ be the maximal torus of $K_{1}$ generated by $i a_{1}$. Then it leaves the Poisson structure $\pi$ on $P_{1}$ invariant (Theorem 4.13). Let $T$ be the subtorus of $T_{1}$ generated by $\mathfrak{t}=i \mathfrak{a}$. Again by Theorem 4.13, the moment map of the $T$-action on $P_{1}$ is given by

$$
J_{T}: P_{1} \longrightarrow \mathfrak{a}: p_{1}=k_{1} a_{1} n_{1} \longmapsto l_{\mathfrak{a}}\left(\log a_{1}\right),
$$

where $l_{\mathfrak{a}}$ means the orthogonal projection from $\mathfrak{a}_{1}$ to $\mathfrak{a}$ with respect to the Killing form of $\mathfrak{g}_{1}$. If we use $\mathfrak{a}_{0}$ to denote the orthogonal complement of $\mathfrak{a}$ in $\mathfrak{a}_{1}$, and set $A_{0}=\operatorname{expa_{0}}$. Then $A_{1}=A A_{0}$, and the moment map for the $T$-action on $P_{1}$ can be regarded as

$$
J_{T}: P_{1} \longrightarrow A: p_{1}=k_{1} a a_{0} n_{1} \longmapsto a .
$$

It is also clear that $J_{T}$ is $\tau$-invariant.

Let $a \in A$, and restrict the $T$-action and the moment map $J_{T}$ to the symplectic leaf $\mathscr{O}_{a, 1}$ of $P_{1}$ through $a$. By Theorem 3.2, the image of $\mathscr{O}_{a}$ under $J_{T}$ is the convex hull of of the image under $J_{T}$ of the fixed point set of $T$ in $\mathscr{O}_{a}$. Since $A$ is a maximal abelian subspace in $P$, any point in $P$ that is fixed by $T$ must lie in $A$; thus the fixed point set of $T$ in $\mathscr{O}_{a}$ is just the intersection of $\mathscr{O}_{a}$ and $A$, which is well known to be the orbit of the relative Weyl group $W=W(K, A)$ through $a$. Since $J_{T}$ restricts to the identity map on $A$, it follows that the image of $\mathscr{O}_{a}$ under $J_{T}$ is exactly the convex hull of the Weyl group orbit $W \cdot a$, which is precisely the statement of the nonlinear convexity theorem (Theorem 2.2) for $G$.

\section{ACKNOWLEDGMENT}

The first author would like to thank her thesis advisor Professor Alan Weinstein for guiding her into the theory of Poisson Lie groups. We owe him many ideas that are essential to the result of this paper. We would also like to thank Professors Hermann Flaschka, Viktor Ginzburg, Franco Magri, Jerry Marsden, Alexey Reyman, and Ping Xu for useful discussions. Special thanks to Professor Bertram Kostant for mentioning the problem to us, to MSI of Cornell University for its hospitality during our stay there in December 1989, and to Professor Victor Kac for bringing reference [Co-Kc] to our attention. 


\section{REFERENCES}

[Ay] M. F. Atiyah, Convexity and commuting Hamiltonians, Bull. London Math. Soc. 14 (1982), $1-15$.

[Co] J. Conn, Normal forms for smooth Poisson structures, Ann. of Math. (2) 121 (1985), 565593.

[Co-Kc] C. de Concini and V. G. Kac, Representations of quantum groups at roots of 1, Operator Algebras, Unitary Representations, Enveloping Algebras, and Invariant Theory, Actes du Colloque en l'honneur de Jacques Dixmier (A. Connes, M. Duflo, A. Joseph, and R. Rentschter, eds.), Progr. Math., Birkhäuser, 1990.

[C-K-P] C. de Concini, V. G. Kac, and C. Procesi, The quantum coadjoint action, preprint, 1991.

[Da-So] P. Dazord and D. Sondaz, Groupes de Poisson affines, Proc. Seminaire Sud-Rhodanien de Geometrie, MSRI series, Springer-Verlag (to appear).

[Dr1] V. G. Drinfel'd, Hamiltonian structures on Lie groups, Lie bialgebras and the geometric meaning of the classical Yang-Baxter equations, Soviet Math. Dokl. 27 (1) (1983), 68-71.

[Dr2] _ Quantum groups, Internat. Congr. Math. (Berkeley, 1986), Vol. 1, Amer. Math. Soc., Providence, RI, 1987, pp. 789-820.

[Du1] J. J. Duistermaat, Convexity and tightness for restrictions of Hamiltonian functions to fixed point sets of an antisymplectic involution, Trans. Amer. Math. Soc. 275 (1983), 412-429.

[Du2] _- On the similarity between the Iwasawa projection and the diagonal part, Soc. Math. France Mem. (N.S.) 15 (1984), 129-138.

[Gz] V. L. Ginzburg, private communication, 1990.

[Gu-St] V. Guillemin and S. Sternberg, Convexity properties of the moment map, Invent. Math. 67 (1982), 491-513.

[He] S. Helgason, Differential geometry, Lie groups, and symmetric spaces, Academic Press, New York, 1978.

[Ho] A. Horn, Doubly stochastic matrices and the diagonal of a rotation matrix, Amer. J. Math. 76 (1954), 620-630.

[KS-Ma] Y. Kosmann-Schwarzbach and F. Magri, Poisson-Lie groups and complete integrability. 1, Drinfel'd bialgebras, dual extensions and their canonical representations, Ann. Inst. $\mathrm{H}$. Poincaré Phys. Theor. (4) 49 (1988), 433-460.

[Kt] B. Kostant, On convexity, the Weyl group and Iwasawa decomposition, Ann. Sci. École Norm. Sup. (4) 6 (1973), 413-455.

[Ku] J. L. Koszul, Crochet de Schouten-Nijenhuis et cohomologie, Astérisque, hors série, Soc. Math. France, Paris (1985), 257-271.

[Lu-We] J-H. Lu and A. Weinstein, Poisson Lie groups, dressing transformations, and Bruhat decompositions, J. Differential Geom. 31 (1990), 501-526.

[Lu1] J-H. Lu, Momentum mappings and reductions of Poisson Lie group actions, Proc. Seminaire Sud-Rhodanien de Geometrie, MSRI series, Springer-Verlag (to appear).

[Lu2] _ Multiplicative and affine Poisson structures on Lie groups, Ph.D. Thesis, University of California, Berkeley, 1990.

[Rs] N. Y. Reshetikhin, Poisson structures for quantum groups at roots of 1, preprint, Harvard Univ., 1990.

[Sc] I. Schur, Über eine Klasse von Mittelbildungen mit Anwendungen auf der Determinanten Theorie, Sitzungsberichte der Berliner Mathematischen Gesellschaft 22 (1923), 9-20.

[STS] M. A. Semenov-Tian-Shansky, Dressing transformations and Poisson Lie group actions, Res. Inst. Math. Sci. 21 (1985), 1237-1260. 
[We] A. Weinstein, The local structure of Poisson manifolds, J. Differential Geom. 18 (1983), 523-557.

[Wo] S. L. Woronowicz, Compact matrix pseudogroups, Comm. Math. Phys. 111 (1987), 613665.

[Xu] P. Xu, private communication, 1990.

Department of Mathematics, Massachusetts Institute of Technology, Cambridge, MASSACHUSETTS 02139

Department of Mathematics, University of California, Santa Cruz, California 95064 